\title{
Estimation of the resonance between forcing and weakly stable oscillations in power systems
}

\author{
Alexey Iskakov ${ }^{1, *}$, Daniil Panasetsky ${ }^{2}$ \\ ${ }^{1}$ V.A. Trapeznikov Institute of Control Sciences, 117997, 65 Profsoyuznaya street, Moscow, Russia \\ ${ }^{2}$ Melentiev Energy Systems Institute, 664033 Lermontov Str. 130, Irkutsk, Russia
}

\begin{abstract}
Using a simple test example, this paper demonstrates that a weakly damped eigen-oscillation of an electric power system can lead to a loss of stability in the presence of its resonance with forcing oscillation. Such resonant interactions can risk the power system security, as confirmed by recent events in the Western American and Nordic power systems. Conventional eigenvalue analysis cannot detect the observed instability due to its non-linear nature. We show that the critical condition for the appearance of this type of instability can be the value of the perturbation energy accumulated in the system over time. To quantify the risk of stability loss, we examine the model of forcing oscillations and the resonance response of power system in a linear approximation and compare it with the simulation results for the two-area Kundur test system.
\end{abstract}

Keywords: resonant modal interactions, forcing oscillations, power system, small-signal stability, interarea oscillations.

\section{Introduction}

Conventional modal analysis evaluates small-signal stability of power systems with respect to some initial disturbance and estimates system eigenvalues and their damping rates. However, when the disturbances are injected into the system regularly, its stability is determined not by the instantaneous dynamics of a single perturbation, but rather by the energy of perturbations accumulated in the system over time.

There are several critically important situations in power systems when the perturbation energy can accumulate in some eigen-modes and cause a resonant reaction in the system. First of all, it has been suggested already in (Vittal et al., 1991) that the stressed power system following a large disturbance exhibits dynamic behavior, not detectable from linear analysis techniques. This suggestion was generally confirmed by the special Task Force established by the Power System Dynamic Performance Committee of IEEE (Sanchez-Gasca et al., 2005). In particular, it was observed in the emergency control that inter-area oscillations with high amplitudes might develop following severe events in the system (Cao et al., 2013). For example, the inter-area low frequency oscillation of $0.224 \mathrm{~Hz}$ occurred due to the loss of two transmission lines in the Western Electricity Coordination Council (WECC) system in the 1996 event. After about one minute, WECC system was broken into four islands with heavy loss of loads (McCalley, 2006). We can suggest that in these cases the initial event produces a large amount of perturbation energy, which is subsequently accumulated in low-damped inter-area oscillations and causes the break of the system.

Another typical situation associated with an accumulation of perturbation energy in power systems are the resonances between inter-area and forced oscillations. These events became observable rather recently due to the installation of real-time detection systems. The forced oscillations can be injected in power systems from cyclic loads or mechanical aspects of generators. When forced oscillations are injected at a frequency close to the frequency of poorly damped interarea mode, the resonance can occur leading to oscillations with high amplitude. These oscillations may have a dangerous effect on generators and power system stability. In a recent event in the Western American power system, a forced oscillation was detected at a frequency close to the frequency $0.37 \mathrm{~Hz}$ of the typical inter-area mode (Sarmadi, Venkatasubramanian, 2016). This oscillation was injected from a hydro generator and originated from the mechanical oscillations of turbine blades. The resonances of forcing oscillations and interarea modes have been recently detected by a real-time detection system in the Finnish power system (Seppänen et al., 2018). In one of the cases, the forced oscillations were caused by misoperating turbine governor in a thermal power plant located in southern Finland and triggered high amplitude oscillations to the tie-lines between Finland and Sweden at $0.3-0.4 \mathrm{~Hz}$. In the other cases, the forced oscillation source was a large load in northern Finland. To the best of our knowledge, a generally accepted theory of the resonances between inter-area and forced oscillations has not been proposed so far.

\footnotetext{
*Corresponding author: isk_alex@mail.ru
} 
Another type of resonances in power systems, which has been investigated intensively in recent years, is the sub-synchronous resonances (SSRs) that arise in a gridconnected wind farms (see references in ( $\mathrm{Du}$ et al., 2019). The series capacitor compensation is an effective means of increasing the total available transfer capability. However, it can cause the SSR, in which electrical energy is increasingly exchanged with the generator shaft system. The SSR can produce the failure of the turbine generator shaft and the subsequent instability of the power system. Of special note is the relatively recent discovery of the phenomenon of the sub-synchronous oscillations (SSO) modal repulsion in power systems integrated with wind turbine generators (WTGs), which can lead to SSR. Such phenomenon can be attributed to the effect of the near strong modal resonance and examination by Ian Dobson explained why the growing SSR may be caused by the near strong modal resonance (Dobson, 2001). In (Du et al., 2018) the modal analysis was used as a way to demonstrate and confirm the impact of the near strong modal resonance. However based on the currently available theory of such resonance, formation of the SSO modal repulsion in the SSR cannot be explained fully.

Although the techniques of mitigation the SSR for conventional turbine generator systems are well established, the SSRs for wind turbine generators originate differently and include many new factors, such as grid interfacing configuration, large number of generators of small power, the specific electronic converters, and variation in the wind speed (Virulkar, Gotmare, 2016). A number of incidents associated with SSR in a grid-connected wind farms were recoded in recent years. Among them are the incidents in the power system ERCOT of Texas (2009), in Guyuan (2012), and Hami (2015) power systems in China. The SSR arising in the wind farms are currently being studied intensively and there is no generally accepted theory of these resonances (Shair et al., 2019).

The mentioned resonances phenomena are crucially important in modern power systems. Unfortunately, the conventional eigenvalue analysis cannot detect the observed instabilities due to their non-linear nature. As an attempt to provide an alternative theory, in this paper we consider a model of the resonance response of a linear system. Assuming that the stability in resonant cases is related to the magnitude of the perturbation energy accumulated in the system over time, we propose a general form of "resonance" stability criteria for the small-stability analysis of power systems. Using the twoarea four machines Kundur test system (Kundur, 1994), we demonstrate how a formally stable eigen-oscillation of an electric power system can lead to a loss of stability in case of its resonance with a forcing oscillation. We compare the simulation results with the proposed theory.

\section{Resonance in a linear system}

In this section a theory of the resonance in a linear system is examined and a general form of the "resonance" stability criteria is proposed. Consider a linear dynamic system with a forcing oscillation

$$
\dot{\boldsymbol{x}}=\mathrm{A} \boldsymbol{x}+\boldsymbol{b} u(t), \text { where } u(t)=F_{0} \cos (\omega t),
$$

where $\boldsymbol{x} \in \mathbb{R}^{n}$ is state vector, $u(t)$ is a forcing signal, $\boldsymbol{b} \in \mathbb{R}^{n}$ is a forcing profile, $\mathbf{A} \in \mathbb{R}^{n \times n}$ is a real matrix with a simple spectrum that can be represented as

$$
\mathrm{A}=\mathrm{U} \Lambda \mathrm{V}=\left[\boldsymbol{u}_{1}, \boldsymbol{u}_{2}, \cdots, \boldsymbol{u}_{n}\right]\left[\begin{array}{cccc}
\lambda_{1} & 0 & \cdots & 0 \\
0 & \lambda_{2} & \cdots & 0 \\
\vdots & \vdots & \ddots & \vdots \\
0 & 0 & \cdots & \lambda_{n}
\end{array}\right]\left[\begin{array}{c}
\boldsymbol{v}_{1}^{T} \\
\boldsymbol{v}_{2}^{T} \\
\vdots \\
\boldsymbol{v}_{n}^{T}
\end{array}\right]
$$

where $(\cdot)^{T}$ is a transpose operation, the matrices $\mathrm{U}$ and $\mathrm{V}$ are composed from the right $\boldsymbol{u}_{i}$ and left $\boldsymbol{v}_{i}$ eigenvectors, respectively, and normalized such that $\mathrm{UV}=\mathrm{VU}=\mathrm{I}$. By applying to (1) the diagonalizing transformation

$$
\boldsymbol{z}=\mathrm{V} \boldsymbol{x},
$$

the dynamical system is split into separate equations for individual eigenmodes as follows:

$$
\dot{\boldsymbol{z}}=\Lambda \boldsymbol{z}+\mathrm{V} \boldsymbol{b} F_{0} \cos (\omega t)
$$

The spectrum of the matrix A consists of real eigenvalues or pairs of complex conjugate eigenvalues. For each real eigenvalue $\lambda_{i}=\gamma_{i} \in \mathbb{R}$, we obtain from (2) the following equation:

$$
\dot{z}_{i}=\gamma_{i} z_{i}+\boldsymbol{v}_{i}^{T} \boldsymbol{b} F_{0} \cos (\omega t),
$$

which has the following specific solution:

$$
z_{i}(t)=\frac{\boldsymbol{v}_{i}^{T} \boldsymbol{b} F_{0}}{\sqrt{\gamma_{i}^{2}+\omega^{2}}} \cos \left(\omega t+\delta_{i}\right), \operatorname{tg} \delta_{i}=\frac{\omega}{\gamma_{i}}
$$

For each pair of complex conjugate eigenvalues $\lambda_{i}=$ $\gamma_{i}+j \omega_{i}$ and $\lambda_{i}^{*}=\gamma_{i}-j \omega_{i}$, we obtain from (2)

$$
\begin{aligned}
\dot{z}_{i} & =\left(\gamma_{i}+j \omega_{i}\right) z_{i}+\boldsymbol{v}_{i}^{T} \boldsymbol{b} F_{0} \cos (\omega t), \\
\dot{z}_{i *} & =\left(\gamma_{i}-j \omega_{i}\right) z_{i *}+\boldsymbol{v}_{i}^{*} \boldsymbol{b} F_{0} \cos (\omega t) .
\end{aligned}
$$

These equations have the following specific solutions:

$$
\begin{gathered}
z_{i}(t)=\frac{-\boldsymbol{v}_{i}^{T} \boldsymbol{b} F_{0}}{2}\left(\frac{e^{j \omega t}}{\gamma_{i}+j \omega_{i}-j \omega}+\frac{e^{-j \omega t}}{\gamma_{i}+j \omega_{i}+j \omega}\right), \\
z_{i *}(t)=z_{i}^{*}(t)
\end{gathered}
$$

Let the spectrum of the matrix A contains $p$ pairs of complex conjugate eigenvalues and $r$ real eigenvalues. Let us go back to the original state variables using the transformation $\boldsymbol{x}=\mathrm{U} \boldsymbol{z}$ and substite here the obtained solutions (3) and (4)

$$
\begin{aligned}
x_{k}(t)=-\sum_{i=1}^{p} \operatorname{Re} & \left\{u _ { i } ^ { k } \boldsymbol { v } _ { i } ^ { T } \boldsymbol { b } F _ { 0 } \left(\frac{e^{j \omega t}}{\gamma_{i}+j \omega_{i}-j \omega}\right.\right. \\
& \left.\left.+\frac{e^{-j \omega t}}{\gamma_{i}+j \omega_{i}+j \omega}\right)\right\} \\
& +\sum_{i^{\prime}=1}^{r} \frac{\boldsymbol{v}_{i^{\prime}}^{T} \boldsymbol{b} F_{0}}{\sqrt{\gamma_{i^{\prime}}^{2}+\omega^{2}}} \cos \left(\omega t+\delta_{i^{\prime}}\right)
\end{aligned}
$$


When there are several weakly stable eigen-oscillations in system (1), the forcing of the state variable $x_{k}$ in (5) in the neighborhood of resonant frequencies is determined by the resonant terms, i.e.,

$$
x_{k}(t) \approx \sum_{\substack{i: \lambda_{i}-\text { weakly } \\ \text { stable }}} \frac{c_{i} \cdot \cos \left(\omega t+\delta_{i}\right)}{\sqrt{\gamma_{i}^{2}+\left(\omega_{i}-\omega\right)^{2}}},
$$

where $c_{i}$ are some constants. The amplitude of $x_{k}$ has characteristic peaks in the vicinity of resonant frequencies. When the frequency of forced oscillations falls into these areas, a system stability violation caused by nonlinear effects of different nature can occur.

A general form of the stability condition in a resonance area should be the limitation of the norm of perturbation of some critical system variables (voltages, currents, frequencies, etc.) denoted further by index $l$, which in the linear approximation can be expressed as:

$$
y_{l}(t)=\sum_{k} \alpha_{k l} x_{k}(t)
$$

Then the "resonance" stability condition can be formulated as

For all $t$ and $l$ :

$$
\left\|\sum_{k} \alpha_{k l} x_{k}(t)\right\| \leq C_{C R I T}^{(l)},
$$

where $C_{C R I T}^{(l)}$ are the coefficients that characterize some critical levels, $x_{k}(t)$ are obtained in (5), and $\|\cdot\|$ is a some kind of norm. In general, these conditions should complement the conventional stability constraints on damping rate of eigen-oscillations.

\section{Experiment with Kundur test system}

In this section, we show in the experiment with the Kundur test system that a formally stable eigenoscillation can lead to a loss of stability in case of its resonance with a forcing oscillation. We also verify that the asymptotic equality (6) holds in the vicinity of the resonant frequency. The model parameters are given in the textbook (Kundur, 1994). The scheme of the experiment is shown in Figure 1.
A perturbation of the automatic voltage regulator (AVR) controller setting point was chosen as a forced oscillation with an amplitude $\Delta U$ in the frequency range from $0.3 \mathrm{~Hz}$ to $1.2 \mathrm{~Hz}$. The perturbation in the electric power of the G3 generator was observed as an output signal.

Figure 2 shows the power swings of generators produced by a harmonic perturbation in the AVR controller set point of $10 \%$. This forced oscillation leads to power swings of the generators G3-G4 of $300 \mathrm{MW}$ (3 p.u.) accounting for about $40 \%$ of the total active power of the system. Clearly, such swings in any real system would lead to a loss of stability due to generator tripping. However, in our case, we use a simple linear model, which is not able to predict specific nonlinear effects leading to generator tripping and the loss of stability.

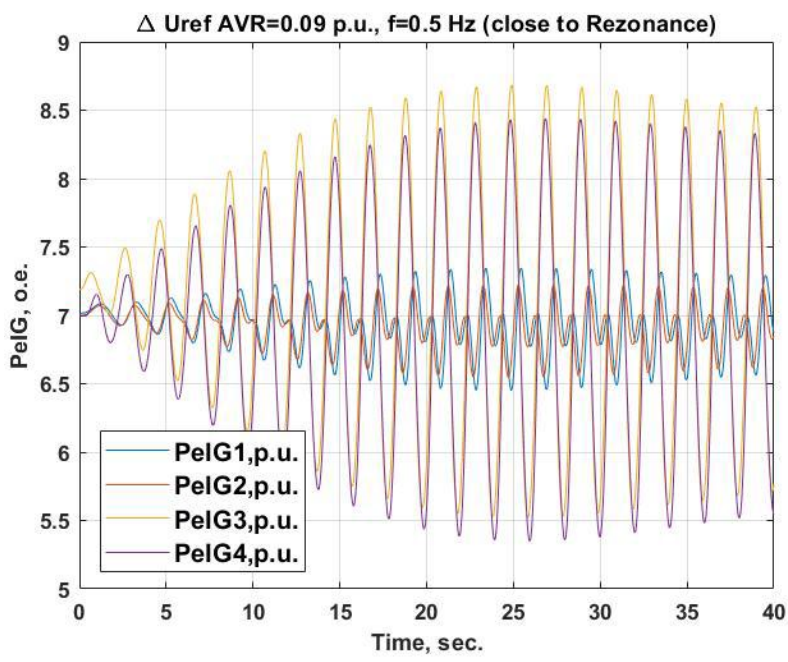

Fig. 2. Power swings of generators G1-G4 produced by a harmonic forced oscillation in the AVR controller set point of $10 \%$.

At the same time, the model in use allows one to predict the shape of the system resonance response. Figure 3 shows the electric power perturbations of G3 generator obtained in simulation and predicted by formula (6) with three resonant terms as a function of an input frequency. One can see that the shape of the resonance curve is fairly well approximated by formula (6).

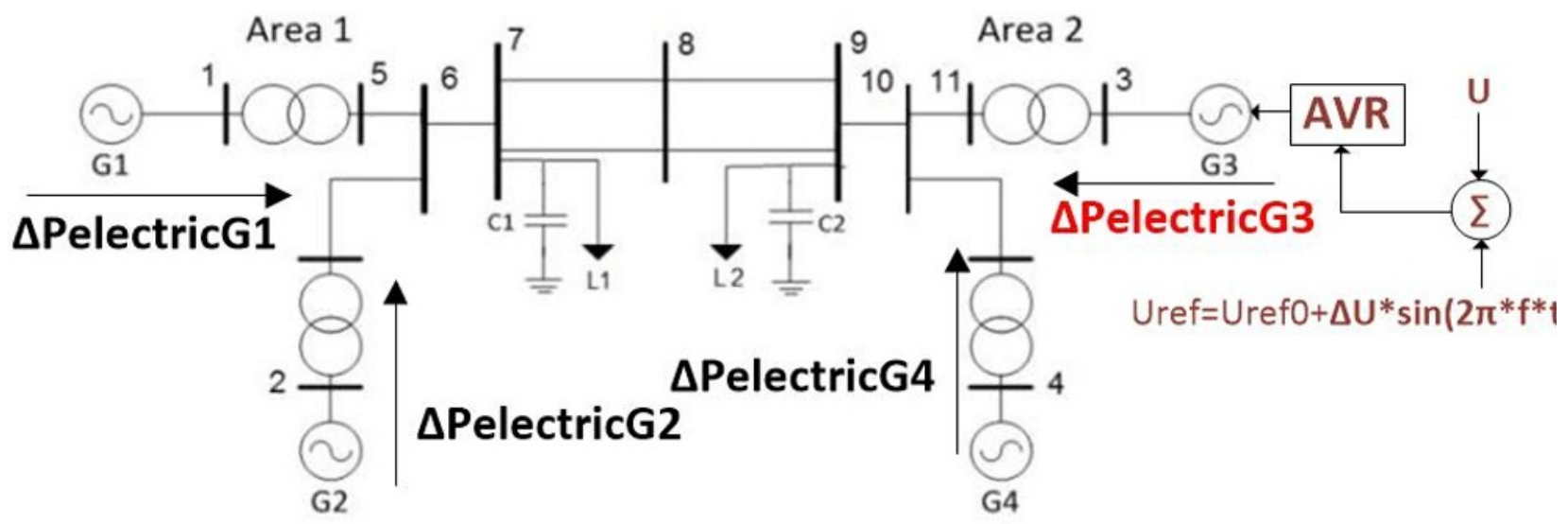

Fig. 1. The scheme of experiment with the two-area Kundur test power system. 

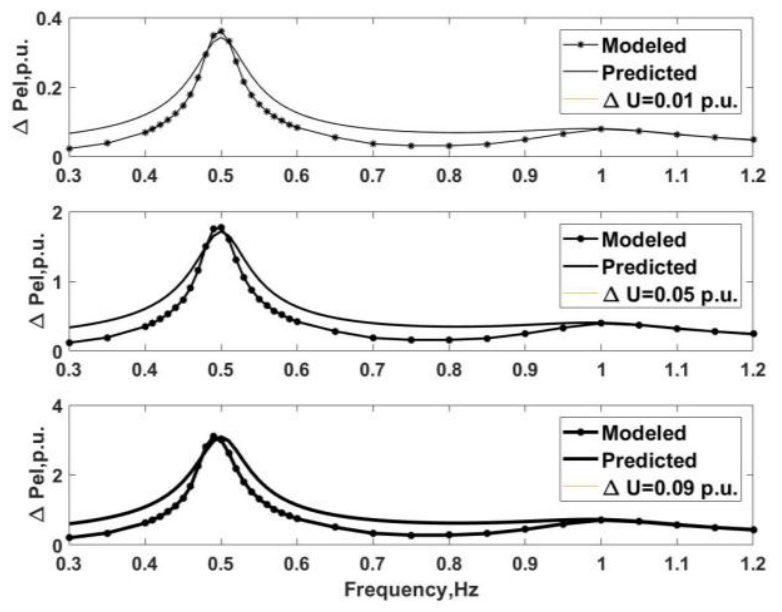

Fig. 3. Electric power perturbations of G3 generator predicted by formula (6) and obtained in simulation as a function of an input frequency $(\mathrm{Hz})$.

\section{Conclusion}

Conventional eigenanalysis can estimate the stability of power systems incorrectly when perturbation energy is accumulated in eigen-modes over time. However, these situations are critically important in modern power systems. In this paper we use the two-area Kundur test system to demonstrate how a formally stable eigenoscillation in power system can lead to a loss of stability in case of its resonance with a forcing oscillation. We also consider a theory of resonance in a linear system and propose a general form of the stability conditions at resonances, which should complement the conventional stability constraints on damping rates of eigenoscillations. This theory, at a first approximation, correctly describes the resonance response of the power system in frequency. At the same time, it does not allow the detection of specific nonlinear effects that lead to a loss of stability. Although there were proposed some more developed theories to describe the effects of resonances in power systems (Yadykin et al., 2016; Vassilyev et al., 2017; Du et al., 2019), there is no generally accepted theory so far. Therefore, a novel theory and methods are urgently required to describe and estimate resonance effects observed in modern power systems.

\section{Acknowledgment}

This work was supported by the Russian Science Foundation, project no. 19-19-00673.

\section{References}

1. V. Vittal, N. Bhatia, A.A. Fouad Analysis of the inter-area mode phenomenon in power systems following large disturbances, IEEE Trans. Power Systems. 6(4), (1991)
2. J.J. Sanchez-Gasca, V. Vittal, M.J. Gibbard, et al. Inclusion of higher order terms for small signal (modal) analysis: committee report-task force on assessing the need to include higher order terms for small-signal (modal) analysis, IEEE Trans. Power Syst., 20(4), 1886-1904, (2015)

3. J. Cao, W. Du, H. Wang, Z. Chen, H.F. Li, A Novel Emergency Damping Control to Suppress Power System Inter-Area Oscillations, IEEE Trans. Power Syst., 28(3), 3165-3173, (2013)

4. I. Dobson, Strong Resonance Effects in Normal Form Analysis and Subsynchronous Resonance, in Proc. Bulk Power System Dynamics Control, Onomichi, Japan, (2001)

5. W. Du, Y. Wang, H.F. Wang, Q. Fu, Concept of Modal Repulsion for Examining the Subsynchronous Oscillations in Power Systems, IEEE Trans. Power Syst., 33(4), 4614 - 4624, (2018)

6. J.D. McCalley, Transmission Security: Rules, Risks, and Blackouts. [Online]. Available: http://www.ece.cmu.edu/cascadingfailures/OpShrtC rseJDM3.pdf, (2006)

7. S.A.N. Sarmadi, V. Venkatasubramanian, Inter-Area Resonance in Power Systems From Forced Oscillations, IEEE Trans. Power Syst., 31(1), 378386 (2016).

8. J. Seppänen, J. Turunen, A. Nikkilä, L. Haarla, Resonance of Forcing Oscillations and Inter-Area Modes in the Nordic Power System, 2018 IEEE PES Innovative Smart Grid Technologies Conference Europe (ISGT-Europe), (2018)

9. V.B. Virulkar, G.V. Gotmare, Sub-synchronous resonance in series compensated wind farm: A review, Renewable and Sustainable Energy Reviews, 55, 1010-1029, (2016)

10. J. Shaira, X. Xiea, L. Wanga, W. Liua, J. Heb, H. Liuc, Overview of emerging subsynchronous oscillations in practical wind power systems, Renewable and Sustainable Energy Reviews, 99, 159-168, (2019)

11. I.B. Yadykin, D.E. Kataev, A.B. Iskakov, V.K. Shipilov, Characterization of power systems near their stability boundary using the sub-Gramian method. Control Eng. Practice, 53, 173-183, (2016)

12. S.N. Vassilyev, I.B. Yadykin, A.B. Iskakov, et al., Participation factors and sub-Gramians in the selective modal analysis of electric power systems, IFAC-PapersOnLine, 50(1), 14806-14811, (2017)

13. F. Du, Q. Fu, H. Wang, Y. Wang, “Concept of modal repulsion for examining the subsynchronous oscillations caused by wind farms in power systems, IEEE Trans. Power Syst., 34(1), 378-386, (2019)

14. P. Kundur, Power Systems Stability and Control. (McGraw-Hill, 1994) 